\title{
Linx
}

Revue des linguistes de l'université Paris X Nanterre

$57 \mid 2007$

Études de syntaxe : français parlé, français hors de

France, créoles

\section{Répétition et progression en français parlé}

\section{Monique Krötsch}

\section{(2) OpenEdition \\ Journals}

Édition électronique

URL : http://journals.openedition.org/linx/264

DOI : 10.4000/linx.264

ISSN : 2118-9692

Éditeur

Presses universitaires de Paris Nanterre

\section{Édition imprimée}

Date de publication : 1 décembre 2007

Pagination : 37-46

ISSN : 0246-8743

\section{Référence électronique}

Monique Krötsch, « Répétition et progression en français parlé », Linx [En ligne], 57 | 2007, mis en ligne le 15 février 2011, consulté le 15 mai 2020. URL : http://journals.openedition.org/linx/264 ; DOI : https://doi.org/10.4000/linx.264 


\title{
Répétition et progression en français parlé
}

\author{
Monique Krötsch \\ Ludwig Maximilian Universität, Munich
}

\author{
À Wolf-Dieter Stempel,

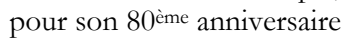 \\ (...) Quelle ombre de tortue \\ Pour l'âme, Achille immobile à grands pas ! ${ }^{1}$
}

\section{Répétition et transcription}

Tous les chercheurs travaillant sur l'oral savent à quel point la lisibilité d'une transcription compte pour l'analyse (voir Gadet 2008). Ils savent aussi que la fidélité au signal sonore, jamais absolue et rarement innocente, n'est ni possible ni souhaitable. D'abord parce que la transcription est le fait d'un transcripteur qui poursuit des buts toujours particuliers (le sociologue n'ayant pas les mêmes que le linguiste); ensuite parce que le produit de la transcription, le transcript ${ }^{2}$, ne transmet pas l'image de l'objet qu'on croit qu'il représente, à savoir le produit de l'activité langagière à l'oral (le texte parlè) : il n'en est que le protocole, la trace verbale, forcément incomplète parce qu'amputée du signal sonore avec ce qu'il contient de verbal : le signifiant prosodique.

\footnotetext{
${ }^{1}$ Paul Valéry, Charmes « Le cimetière marin », v. 125-126.

2 En accord avec Gadet 2008, note 5, je trouve la distinction entre action de transcrire (transcription) et produit (transcript) nécessaire; la graphie <transcript> souligne l'appartenance du produit à la famille des scripta.
} 
Or, l'appréhension visuelle créant une attente visuelle, les stratégies de "lecture » activées par le passage au graphique, au lieu de construire du sens, le délitent, et renvoient au lecteur l'image d'un produit inachevé.

Un demi-siècle après l'irruption des données orales dans l'analyse linguistique et les tentatives pour accorder ce qu'il faut «donner à voir» à ce que l'enregistrement «donne à entendre », la recherche, qui a tiré de l'impossible entreprise un outillage conceptuel permettant de séparer ce qui était jusque là confondu ${ }^{3}$, est en mesure aujourd'hui de prendre en compte ce «décalage sémiotique irrémédiable (Gadet 2008, pour toute la problématique écrit-parlé).

Cependant, identifier les raisons du décalage ne le fait pas disparaittre, et le transcript, au lieu de servir l'analyse, peut la dévoyer. La connexion « français parlé mauvais français » a beau être régulièrement dénoncée, elle n'en est pas moins active. Renforcée par la manipulation graphique de réalisations phoniquement neutres (Gadet 2008, Koch/Oesterreicher 2001), pratiquée de façon sporadique jusqu'à la fin du siècle dernier, elle survit dans la priorité accordée à l'interprétation majoritairement diastratique des phénomènes de l'usage oral.

La répétition, dans la production langagière non planifiée, tombe souvent sous ce type d'explication. Partageant avec quelques autres phénomènes (erreurs, ratages, interruptions, phénomènes d'hésitation, faux départs, bribes...) la propriété d'interrompre le « fil du discours », l'appréhension qu'en donne le transcript est si laborieuse, qu'on est tenté de la cataloguer sans plus d'investigation comme accident de performance ou maladresse du locuteur. Les deux interprétations se prêtant bien à une approche conversationnelle ${ }^{4}$, la méconnaissance de sa matérialité discursive passe inaperçue, moyennant quoi il est rare qu'elle fasse l'objet d'une description strictement linguistique ${ }^{5}$.

C'est précisément de cette matérialité qu'il sera question ici. Après avoir écarté comme principe d'analyse la comparaison écrit-parlé et défini un cadre permettant une approche neutre des faits, je m'interrogerai, de façon utilitaire, sur sa finalité - à ne pas confondre avec l'intentionnalité (à quoi la répétition sert-elle, ou plutôt : que fait-elle dans les textes ${ }^{6}$ ?), en partant des formes attestées dans les transcripts, et en insistant sur la part active qu'elle prend à la progression du texte parlé. Le côté paradoxal de la

\footnotetext{
${ }^{3}$ Rappel terminologique : le transcript est le produit de la transcription, elle-même activité de transcodage. Ce passage au graphique (all. Verscbriftung), résultant du changement de médium (ou canal), se distingue du passage à l'ecrit (all. Verschriftlichung) qui, en opérant sur la conception, est reformulation, réécriture. La distinction code-médium (Söll, 1974) a été décisive.

${ }^{4}$ La problématique «francais parlé » ne se confond pas avec l'analyse conversationnelle; Bronckart $(1997,102)$ souligne que la spécificité des textes parlés peut être saisie indépendamment des contenus (qui sont les mêmes, à l'écrit et à l'oral) à partir de paramètres linguistiques en nombre limité définis préalablement (Bronckart et al. 1985, 74-75).

${ }^{5}$ Comme le souligne Bruno Martinie (2001, 189), le GARS (voir les nombreux articles dans RSFP depuis 1979) fait figure d'exception. L'analyse conversationnelle n'est pas en cause ici. Elle ne peut cependant en aucun cas remplacer l'approche linguistique.

${ }^{6}$ Une des limites de l'analyse du GARS est de prendre l'énoncé comme cadre d'analyse : c'est à mon sens confondre production et produit.
} 
formulation n'est pas dû au hasard; entre immobilité et mouvement, je prends au mot la contradiction : c'est dans l'apparente incompatibilité de ces termes que réside le moteur de la répétition.

\section{Rhétorique et fugacité de l'oral}

Avant de poursuivre, deux mises au point sont nécessaires :

1) Pourquoi aborder la répétition dans l'usage oral par l'appréhension visuelle qu'en donne le transcript, alors qu'on vient de souligner les difficultés inhérentes à celui-ci ? La réponse est simple : parce qu'on ne l'« entend » pas... Sans transcript, on n'y prêterait pas attention ${ }^{7}$. Ce n'est que par une écoute active, répétée, non impliquée (autrement dit, une écoute de transcripteur), qu'on parvient à la saisir, "noir sur blanc $»$.

2) Pourquoi traiter le phénomène à l'oral sans l'envisager par rapport à l'écrit ? La comparaison écrit-parlé, polarisante, appliquée à un phénomène discursif tel que la répétition, ne peut qu'être redondante et confirmer ce qu'on sait déjà, à savoir que la différence écrit-parlé existe. Celle-ci tourne d'ailleurs toujours au désavantage du parlé : mesuré à l'aune de l'écrit, il ne peut être que déficitaire ${ }^{8}$. Enfin, on peut se demander ce qui est effectivement "comparé ». Des textes? Mais le texte parlé, plurisémiologique, surdéterminé contextuellement (Adam 1999), n’est tout simplement pas comparable à un texte écrit, d'où le soupçon : ne serait-ce pas le transcript qui, pris pour le texte parlé, est comparé à un texte écrit ??

Comment décrire sans déclasser? La répétition étant une figure, la rhétorique peut fournir une approche neutre ${ }^{10}$. Dans un dictionnaire de rhétorique assez récent (Molinié, 1992), le rapport entre rhétorique et langage est du reste souligné sans ambiguité. Loin de se répéter par maladresse, les locuteurs " font de la rhétorique » comme Monsieur Jourdain faisait de la prose, sans le savoir, et retrouvent par là même instinctivement la fonction originelle de cet art, à savoir une technique de la parole efficace $^{11}$. Ils appliquent spontanément en situation des stratégies aptes à faire «marcher» leur affaire ('action langagière en cours) pour la mener à bien, à leur avantage naturellement, c'est-à-dire en la faisant exister hors d'eux-mêmes ${ }^{12}$.

\footnotetext{
${ }^{7}$ On pourrait ajouter : sans transcript, pas de «langue parlée » non plus, ce qui souligne la valeur heuristique de la transcription.

${ }^{8}$ Le point de départ de la comparaison étant l'écrit, il ne peut pas en être autrement.

${ }^{9}$ Mais lequel? Le produit du passage à l'écrit du texte parlé (réécriture) ou n’importe quel texte écrit ?

10 Bien que la répétition ne soit pas spécifique à l'oral (la définition qui suit englobe l'écrit aussi bien que l'oral), elle s'y manifeste de façon spécifique, en combinaison avec les «accidents» de performance évoqués plus haut (interruptions etc.). Dans ce contexte, elle fait effectivement figure de « réparation » (Martinie 2001).

${ }^{11}$ L'importance prise au cours des siècles par l'axiologie a pu donner l'impression que la rhétorique avait pour but de porter des jugements sur la qualité esthétique de textes écrits.

12 Krötsch $(1999,34-38)$ : une locutrice, partie sur une visée fausse, « sort » du communicatif jusqu’à ce qu'elle ait réussi à la mettre dans le bon sens.
} 
La définition de Molinié, en établissant clairement un cadre d'analyse linguistique, laisse pressentir le rôle actif de la répétition :

La répétition est une figure de type microstructural spécialement à l'œuvre dans les figures d'élocution et même de construction. (... Elle) constitue la plus puissante de toutes les figures : (...) dès que l'on passe à la question des contenus textuels, on déborde la limite microstructurale propre à la figure qui peut dès lors servir de base formelle (...) à des figures macrostructurales. La reprise qui définit la répétition peut toucher le son (la lettre, la syllabe), le mot, le groupe de mots, la phrase, le paragraphe, le texte entier - ou encore l'idée. (Ibid., pp. 292-293 ; c'est moi qui souligne).

Après avoir différencié les cas de répétition d'après le rapport synonymique qu'entretiennent le terme répété et sa reprise, l'auteur ajoute :

(...) On peut voir la répétition, éclatant hors de sa vraie nature, à l'œuvre comme principe productif dans les reprises ou les étalements de contenus des figures macrostructurales d'amplification. (...). La répétition est donc la figure qui conditionne tout discours.

J'essayerai dans ce qui suit de montrer de quelle façon se manifeste ce « principe productif ».

\section{La répétition comme support}

La répétition est le support par excellence de l'activité langagière à l'oral. Elle garantit à la fois la progression, la cohésion et l'interprétabilité du texte parlé :

(1) L1 : (..) s'i(l) postule pour un emploi de magasinier je veux bien mais s’i(l) postule pour un emploi de vendeur $i(l)$ donne pas envie

L2 : donc vendeur on doit arriver comment (J'ai un problème, "le chômage », France 2, 1995).

La reprise vendeur en limitant le cadre d'interprétation du second énoncé augmente la cohésion de l'échange (Stark, 1997).

Associée à des phénomènes de rupture de chaîne, la répétition, perçue ellemême comme rupture, dans l'appréhension visuelle du transcript, assure au contraire la continuité, comme le montre l'exemple suivant:

(2) L1 : (...) il a tenu à prendre quat(re) personnes qui appartiennent à la caste parce que c'est une caste - des maçons des ouvriers du bâtiment qui est sans doute la caste la plus rél

L2 : trois trois [j] en a que trois pa(rce) que

L1 : [j] en a que trois non le quatrième: est: contremaitre donc de toute façon euh: euh: qui fait $\mathrm{i}(\mathrm{ls})$ ne font pas partie de Solidarité et ce et cette caste est la plus RÉactionnaire parmi les ouvriers en : en Pologne (Morel, 1985, 35 ; reproduction de la transcription de : Christine Leroy).

La reprise, en amont de l'interruption, permet, au sens propre du terme, de «renouer » le fil, très exactement (à la syllabe près, l'accent initial sur Réactionnaire soulignant la jonction) là où il a été rompu. 
Reprise rétroactive également, dans l'exemple qui suit, sans interruption toutefois, mais par nécessité d'expliciter un groupe nominal de structure complexe (un financement des Gabonais aux partis français), que, pour des raisons relevant de l'interaction (elle risque à tout instant d'être interrompue), la locutrice jette dans la discussion sous une forme très compacte :

(3) (...) oui c'est-à-dire moi je sais une histoire très drôle que Pierre connait bien un financement des des des Gabonais enfin [sta]dire aux partis français par les Gabonais aux trois partis français aux trois plus grands partis français au moment de quatre-vingt un au moment des élections (...) (Droit de réponse, «La corruption électorale » Antenne 2, 1986).

Dans la mise en grille, le groupe à décompacter (lignes 1-2) est comme un cadre qui se remplit d'une ligne à l'autre (lignes 3-5) par insertion (de droite à gauche) d'un nouvel élément :

$\begin{array}{llll}1 & \text { un financement } & \text { des Gabonais } & \\ 2 & \text { par les Gabonais } & \text { aux } & \\ 3 & & \text { aux trois } & \text { partis français } \\ 4 & & \text { aux trois plus grands partis français }\end{array}$

Consciente de l'ambiguité (ligne 1 : génitif subjectif ou objectif ?), la locutrice souligne la structure actancielle du déverbal financement (ligne $3:$ différenciation CAUSATAIRE - BÉNÉFICIAIRE), bien que l'ambiguïté ait été levée auparavant (ligne 2 : complément d'attribution aux partis...), puis réduit par étapes (lignes 4 et 5) l'extension du BÉNÉFICIAIRE avant de poursuivre l'énoncé resté en suspens.

Malgré sa simplicité grammaticale, l'exemple suivant, pénible à lire, fait problème : à quoi peut servir la répétition ?

(4) c'est vrai que il y a des choses que : il : il faut : euh ff/ comment je pourrais te dire ça :: il faut revendiquer plus fort que certaines choses :: mais enfin :: il faut pas laisser les autres choses aussi à/de côté mais il y a des choses qui doi/qui doivent se revendiquer plus fort :: voilà (Blanche-Benveniste 1988, 56).

Est-ce l'absence de contexte qui rend l'interprétation difficile? Manque, en effet, l'arrière-plan, le contenu de la discussion qui précède, ce qui mettrait des référents derrière les choses, inscrirait (4) dans une thématique connue, balisée par du lexique (revendiquer ouvre une première piste...). Le problème pourtant n'est pas l'absence de contexte mais la forme de (4) en tant que texte, validé, de plus, par le ponctuant final voilà, qui suit une pause longue, sans que l'allocutaire (te) réclame un supplément d'information. De toute évidence, le locuteur est satisfait. C'est donc qu'il a « réussi ». Mais en quoi ? 
Voilà, en donnant un signal non ambigu de finitude ${ }^{13}$, fait de ce fragment un espace discursif à l'intérieur duquel se construit une cohérence qui lui est propre, et par rapport à laquelle toutes les actions langagières sont interprétables. Cette cohérence, qui relie (4) à tous le(s) contexte(s) linguistique(s) et non linguistique(s) qui l'ont rendu possible (avec tout ce que cela suppose d'implicite), a besoin d'être reconstruite. Au point de vue formel, on peut ramener (4) à trois assertions :

(i) il y a des choses qu'il faut revendiquer plus fort que certaines choses;

(ii) mais il faut pas laisser les autres choses de côté ;

(iii) mais il y a des choses qui doivent se revendiquer plus fort.

La comparaison initiée dans (i) entre des choses (qui constituent le thème de l'échange) et certaines choses (établissant l'importance supérieure des premières sur les secondes), restreinte dans (ii) par le premier mais (les autres choses, qui incluent ou s'identifient aux certaines choses de (i), ne sont pas inimportantes), est reprise dans (iii) avec une modification importante, plus sans que ayant une valeur absolue (un contenu de superlatif avec une forme de comparatif...), ce qui, sans remettre en question la restriction de (ii), met les choses « tout court » à l'abri d'une comparaison. En désamorçant l'implication qu'on peut tirer de (i), (iii) confirme la suprématie des choses tout court, en ayant l'air d'établir une échelle des valeurs valable pour toutes les choses présentes, à l'exclusion des choses tout court.

Les opérations langagières effectuées dans (4) portent sur la seule mise en relation de notions qui restent abstraites. Elles ne sont que minimalement différenciées, par les indéfinis adnominaux des, certaines, autres, et représentées lexicalement sous la forme la plus vide possible, choses, qui fonctionne comme un joker. (4), qui conclut la discussion, décompose la mise en relation en trois phases. L'intrication des arguments, des mouvements de pensée contradictoires, est telle (planification et production langagières sont synchrones), que la tâche «mise en relation" ne fait l'objet ni d'un ancrage énonciatif commun, ni d'une textualisation intégrant les trois assertions en une « période » unique.

Cette séparation entre notions et mise en relation des notions (Bronckart 1985) est une des caractéristiques les plus marquantes de l'usage oral et contribue largement à lui donner son caractère "agrégatif» (Ludwig 1986). Elle n'est praticable (et effective) que si le locuteur s'appuie sur la seule technique qui permette l'atomisation des contenus et la séparation des tâches sans que les locuteurs «perdent le fil »: la répétition. En faire une erreur de planification est aberrant : elle rend au contraire la planification à court terme possible dans la production et est en cela bien adaptée à la relance du texte parlé, foncièrement ouvert.

\footnotetext{
${ }^{13}$ La finitude est le bornage extérieur d'une suite d'événements linguistiques qui forment de ce fait une « unité »; l'achèvement définit l'unité de l'intérieur, d'après des critères formels, par la saturation morpho-syntaxique.
} 


\section{La dynamique de la reprise}

Ce serait pourtant réduire le potentiel de la répétition que de la considérer seulement dans sa fonction de support (qui reste sa fonction basique). Molinié 1992 reconnait dans la reprise le "principe productif» de la répétition. Chaque reprise, même à l'identique (ex. (1) et (2)), apporte sa modification (ne serait-ce que la différence de fonction du terme répété). La dynamique réside dans la rétroactivité de la reprise, qui intègre ce qu'il faut de contexte en amont (cohésion renforcée) pour « rebondir ». Soit l'exemple :

(5) ils ont des appareils pour pour vraiment pour savoir d'où ça vient pour déterminer vraiment la cause il y a des appareils (Blanche-Benveniste et al. 1979, 168).

La mise en grille (5a) montre que non seulement (5) est très lisible :

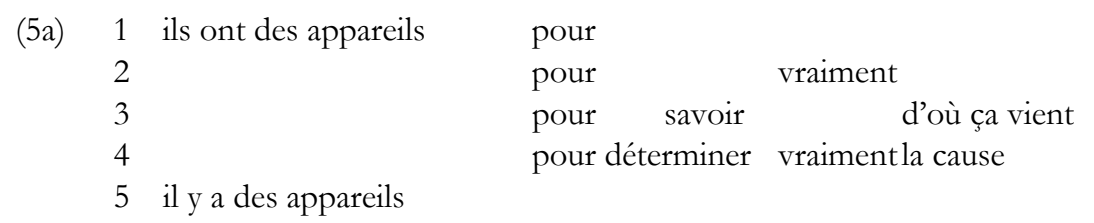

mais que chaque «retour à la ligne» ajoute, ou modifie un élément. L'ajout se fait horizontalement, aussi bien droite-gauche que gauche-droite, lignes 1-3; la modification, verticalement (reprise de contenu des lignes supérieures). On a répétition de mots (pour, vraiment, appareils) et de contenu sémantique (déterminer $=>$ savoir et la cause $=>$ d'où ça vient). Si on considère la modification apportée par les lignes $4-5$, on voit qu'elle ne concerne pas que les mots, mais l'ensemble formé par les lignes 1-3, mis dans un autre espace communicatif. La différence entre les deux espaces se lit dans les formes linguistiques : ils non référencié (ligne 1), ça (ligne 3), indiciel (« tout ce qu'il y a là » ou «tout que j'ai dit avant»), qui font place respectivement au dispositif de prédication neutre il y a (ligne 5), tandis que ça, dont le contenu, trop vague ou trop vaste, n'est pas spécifiable en complément déterminatif de la cause (seule place syntaxique disponible) est abandonné.

(5a) montre le locuteur en train d'agir : il ne cherche pas ce qu'il veut dire : il explore l'espace communicatif, s'essaye dans l'un, puis dans l'autre. La grille propose non pas un énoncé, mais deux énoncés potentiels, différenciables linguistiquement sur la base des pronoms, et communicativement par leur positionnement entre les pôles «immédiat » et « distance » : le premier énoncé est du côté de l'immédiat, encore dans la production, la formulation ; le second, du côté de la distance, de la reformulation abstraite. Ils forment ensemble ce que Culioli appelle une «famille paraphrastique » (Culioli 1987 (1990-2000, tome 1, 26). 
Dernier point, (5a) rend visible la structure « en miroir» (Blanche-Benveniste $e t$ al. 1979, 183 et s.) des deux énoncés potentiels (i) et (ii) ${ }^{14}$ :

(i) ils ont des appareils

(ii) pour déterminer vraiment la cause

pour savoir vraiment d'où ça vient il y a des appareils

La répétition avec chiasme est la structure par excellence d'ouverturefermeture, faisant de ce qu'elle inclut une unité discursive, qui, reconnue comme telle, contribue à la structuration du texte. Dans les échanges consensuels, la combinaison peut se répéter elle-même tout en mutant (elle est «filée ») au long du texte ; elle est comme un motif musical qui rythme le texte et que les locuteurs se partagent ${ }^{15}$. Ce motif définit un autre espace de cohérence, englobant les autres : celui de la complicité du groupe. La place manquant ici pour un exemple long, j'illustre la combinaison répétition et chiasme, par ce joli exemple de gestion commune par deux adolescentes :

(6) L1 : on s'inquiétait pas pour les études on on y allait comme ça on faisait en cours on faisait

L2: on fait que $(. .$.$) on faisait des bêtises$

L1 : on faisait que des bêtises en cours toutes les deux (...) (Andersen \& Thomsen, 2004, 227-228. Les soulignements sont produits simultanément).

\section{Le temps « éclaté "}

Certaines catégories linguistiques, comme le temps, l'aspect, semblent fournir à la répétition une matière idéale. On les voit éclater littéralement dans l'usage oral, notamment quand il s'agit de récit :

(7) (...) parce qu'il est pas jeune maintenant hein alors pendant la guerre il avait notre âge maintenant il a notre âge (Blanche-Benveniste 1985, 121).

L'organisation globale de la temporalité n'est pas seulement difficile, mais impossible, dans un texte ouvert, dont la planification et la production sont synchrones, quand les événements relatés ne sont pas dans le présent du locuteur. Pour (7), la reprise de avoir le même âge au présent, plus que l'intensification du sentiment du temps qui passe, me semble séparer définitivement les deux moments, effaçant la continuité qui les relie.

Les reprises multiples d'un verbe à des aspects différents décomposent les procès suivant la même logique de séparation que l'exemple (4). On a deux cas de figures : soit c'est le déroulement temporel qui est décomposé en phases successives (du type : c'est à faire, ça se fait, c'est fait, c'est plus à faire) sans qu'il y ait continuité entre elles; soit, c'est le procès qui, mis hors du temps, éclate dans la multiplicité des points

14 L'effet de miroir devrait être validé prosodiquement, comme me le fait remarquer Paul Cappeau. N'ayant pu vérifier auditivement (ii) (une ou deux unités ?), il est difficile de décider.

15 Aspect structurant qui ressort bien dans l'analyse de conversations longues. Voir à ce sujet Tannen 1987 (je remercie Paul Cappeau d'avoir attiré mon attention sur cet article) qui examine le fonctionnement de la répétition à la fois dans la production, la compréhension, la cohésion et l'interaction, tout en prenant en compte le lexique, la syntaxe et la prosodie. 
de vue d'où on peut le considérer. Dans le récit «L'accident» (dont je reproduis dans (8), dans l'ordre où elles surviennent, les constructions de voir), la locutrice, qui revit en le rapportant l'évènement traumatique auquel elle a finalement réchappé (elle n'est pas passée sous la voiture...), n'en finit pas de se passer, en short cuts, le film de sa frayeur :

(8) (...) je me suis vue la voiture dessus (...) j’ai vu le dessous de la voiture (...) je me voyais dessous $(\ldots)$ je l'ai vue sur moi $(\ldots)$ tu te vois dessous quoi $(\ldots)$ je me la suis vue dessus (...) j'ai eu le temps de voir le dessous (...) (Blanche-Benveniste et al. 2001, 127-131).

À l'issue de ce tour d'horizon, une des fonctions centrales de la répétition dans les textes parlés me semble être la fragmentation. Figure contradictoire, les tâches auxquelles elle sert de support ne le sont pas moins : séparer pour rapprocher, effacer pour tenir en mémoire ${ }^{16}$, disperser pour structurer. Possibilité d'expansion illimitée, la répétition sert à la fois de point d'appui et de repère au locuteur qui s'aventure dans le «pas encore dit » sans lâcher le « déjà dit », celui-ci se raréfiant, peu à peu.

On a identifié plus haut la « réussite » de l'activité langagière comme la capacité à faire exister hors de soi une intention de communication. Dans cette perspective, on comprend mieux l'importance de la fragmentation : elle en est la condition nécessaire. S'approprier la parole, c'est sortir du continu, du fusionnel ; dépasser le geste, ou le cri, inarticulés. Si l'oralité est l'immédiat corporel, la fragmentation est, plus qu'une étape décisive dans la prise de distance, la condition même du discours.

Achille ne rattrapera pas la tortue. Mais il ira beaucoup plus loin.

Munich, mai 2009

\section{RÉFÉRENCES BIBLIOGRAPHIQUES}

ADAm, J. M. (1999) : Linguistique textuelle. Des genres de discours aux textes, Paris, Nathan.

Andersen, H. L., Thomsen, C. (2004) : Sept approches à un corpus. Analyses du francais parlé, Bern, Peter Lang.

BLANCHe-BENVENISTE, C . et al. (1979) : «Des grilles pour le français parlé », RSFP 2, GARS, Aix-en-Provence, 163-206.

BlanCHe-Benveniste, C . (1985) : "La dénomination dans le français parlé: une interprétation pour les "répétitions" et les "hésitations", RSFP 6 (1984), GARS, Aix-enProvence, 109-130.

Blanche-Benveniste, C . (1987): «Syntaxe, choix de lexique et lieux de bafouillage», DRLAV 36-37, Dialogues : du marivaudage à la machine, 123-157.

16 Ce qui correspond à la capacité de tenue en mémoire ( 7 à 10 mots) et de projection (également 7 à 10 mots), permettant les aller-retours sur l'axe syntagmatique en traduction simultanée (Lederer, 1986). 
BlanCHE-Benveniste, C. (1988) : «La notion de contexte dans l'analyse syntaxique des productions orales. Exemples des verbes actifs et passifs ", RSFP 8, GARS, Aix-enProvence, 39-57.

Blanche-Benveniste, C. (2001) : Choix de textes de français parlé, Paris, Honoré Champion.

BronCKART, J. P. (Ed.) (1985) : Le fonctionnement des discours. Un modèle psychologique et une méthode d'analyse, Neuchâtel-Paris, Delachaux \& Niestlé.

Bronckart, J. P. (1997) : Activité langagière, textes et discours. Pour un interactionnisme socio-discursif, Paris-Lausanne, Delachaux \& Niestlé.

Culioli, A. (1983) : « Pourquoi le français parlé est-il si peu étudié ?», RSFP 5, GARS, Aix-enProvence, 291-300.

Culioli, A. (1990-2000) : Pour une linguistique de l'énonciation. Opérations et représentations, Gap-Paris, Ophrys.

Gadet, F. (2008) : «L'œil et l'oreille à l'écoute du social», in Données orales. Les enjeux de la transcription, Bilger (dir) Les cahiers no 37, Presses universitaires de Perpignan, 35-48.

Koch, P., Oesterreicher, W. (2001) : «Langage parlé et langage écrit » in Holtus et al. (Eds) LRL I, 2, Tübingen, Max Niemeyer Verlag, 584-627.

KRÖTSCH, M. (1999) : «Problèmes de mise en chaîne en français parlé : „Ruptures” syntaxiques ou indices de gestion réussie ? ", RJb 49 (1998), 30-40.

LEDERER, M. (1986) : « Implicite et explicite », in Interpréter pour traduire, Paris, Didier Érudition.

LERoY, C. (1985) : «La notation de l'oral », in Morel, M.A. L'oral du débat (= Langue française 65), Paris, Larousse, 6-16.

Ludwig, R. (1986) : «Mündlichkeit und Schriftlichkeit. Felder der Forschung und Ansätze zu einer Merkmalsystematik im Französischen“, RJb 37, 15-45.

MARTinie, B. (2001) : «Remarques sur la syntaxe des énoncés réparés en français parlé. », in RSFP 16, 189-206.

MoliniÉ, G. (1992) : Dictionnaire de rhétorique, Paris, Le Livre de Poche.

OesterReicher, W. (1993) : “'Verschriftung' und 'Verschriftlichung' im Kontext medialer und konzeptioneller Schriftlichkeit » in Schäfer, U. (Ed.) Schriftlichkeit im frühen Mittelalter, Tübingen, 267-292.

SöLL, L. (31985) : Gesprochenes und geschriebenes Französisch, Berlin, Erich Schmidt (première édition: 1974)

STARK, E. (1997) : Voranstellungsstrukturen und "topic"-Markierung im Franæösischen. Mit einem Ausblick auf das Italienische, Tübingen, Narr.

STEMPEL, W. D. (1987) : "Die Alltagserzählung als Kunst-Stück », in Erzgräber/ Goetsch (Eds.), Mündliches Erzäblen im Alltag, fingiertes mündliches Erzäblen in der Literatur (ScriptOralia 1), Tübingen, Gunter Narr Verlag, 105-135.

STEMPEL, W. D. (1994) : «Stylistique et interaction verbale » in Molinié \& Cahné Qu'est-ce que le style?, Paris, PUF, 313-330.

Tannen, D. (1987) : «Repetition in Conversation: Toward a Poetics of Talk », Language 63, 574-605. 